\title{
Editorial
}

\section{Econophysics, Statistical Mechanics for Financial Applications, and Financial Mathematics}

\author{
Doojin Ryu ${ }^{1}$ and Kiseop Lee ${ }^{2}$ \\ ${ }^{1}$ College of Economics, Sungkyunkwan University, Seoul 03063, Republic of Korea \\ ${ }^{2}$ Purdue University, 610 Purdue Mall, West Lafayette, IN 47907, USA \\ Correspondence should be addressed to Doojin Ryu; doojin.ryu@gmail.com
}

Received 30 May 2016; Accepted 30 May 2016

Copyright (C) 2016 D. Ryu and K. Lee. This is an open access article distributed under the Creative Commons Attribution License, which permits unrestricted use, distribution, and reproduction in any medium, provided the original work is properly cited.

Econophysics, statistical mechanics, and financial mathematics are interdisciplinary study that combines economics, statistical physics, stochastic analysis, stochastic processes, optimization, numerical methods, and quantitative finance. This special issue presents five high-quality researches in this interdisciplinary area.

A. Ishikawa et al. analytically show that the logarithmic average sales of firms first follow power-law growth and subsequently follow exponential growth, if the growth-rate distributions of the sales obey the extended-Gibrat's property and law. They analytically show that the parameter of the extended-Gibrat's property is identical to the powerlaw growth exponent and that it decides the parameter of the exponential growth. Y. H. Shin and H.-S. Lee propose a regime switching model of schooling choice as a job search process. They adopt a two-state Markov process and the derived coupled Bellman equations and suggest some numerical examples in this framework. D.-L. Sheng focuses on a reserve process with dynamic income to study the reinsurance-investment problem for an insurer under Vasicek stochastic interest rate model. C. Nie and X. Jin effectively predict the long-term time series data to schedule an investment strategy and obtain higher profit, considering that a stock price is a typical but complex type of time series data. They argue that their prediction method on the longterm stock price dynamics can be more precise and prevent the development of cumulative errors compared to the other methodologies. C. Xie et al. test the existence of systematic risk contagion within the Chinese interbank market with a particular data set from 2005 to 2013. By building the networks of the Chinese interbank market for each year and using the measure of mutual information, they quantitatively detect the changes of interbank market networks and observe that the correlations between banks become increasingly tighter in recent years.

By compiling these interdisciplinary and quantitative papers, the editors hope to enrich the readers and academic researchers knowledge with respect to the econophysics, statistical mechanics, and quantitative economics and finance.

Doojin Ryu Kiseop Lee 


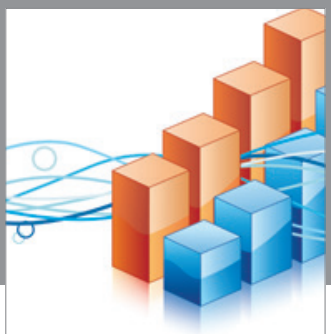

Advances in

Operations Research

vatem alat4

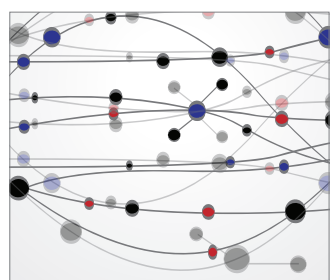

\section{The Scientific} World Journal
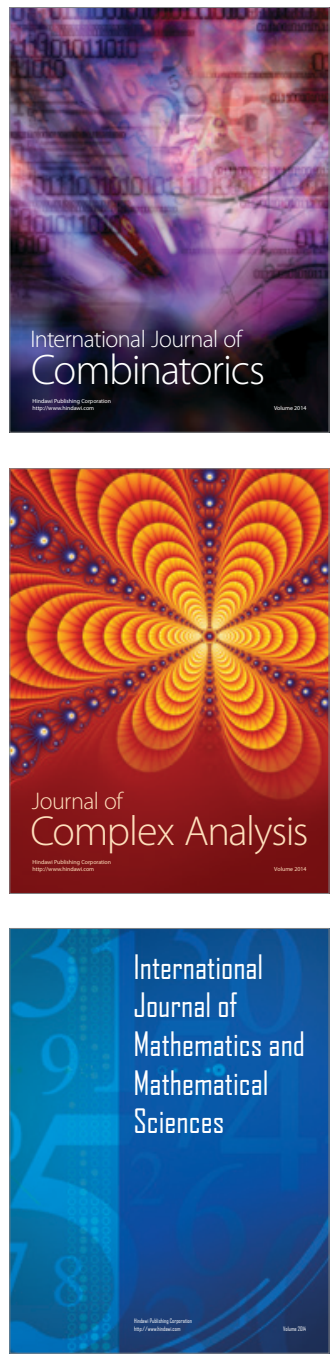
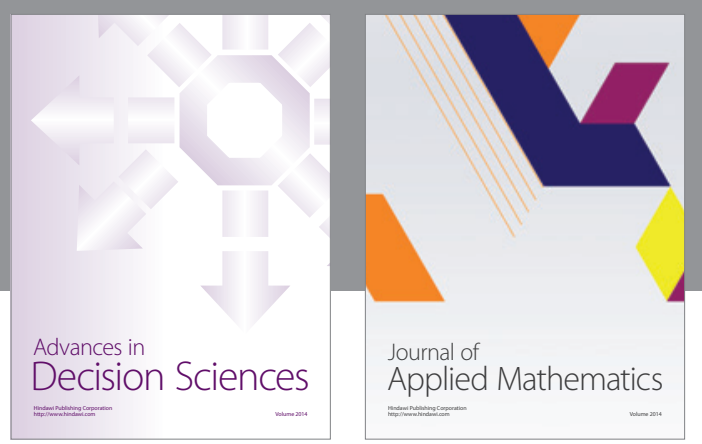

Algebra

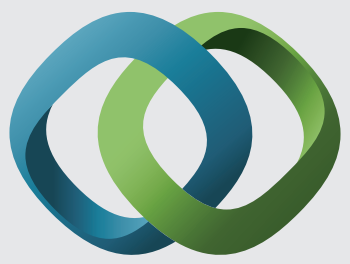

\section{Hindawi}

Submit your manuscripts at

http://www.hindawi.com
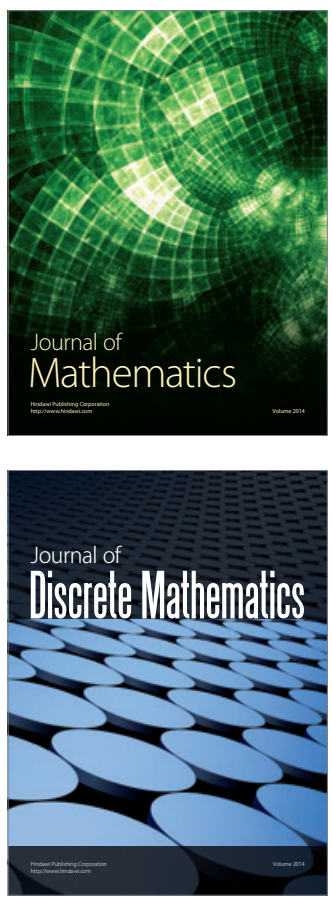

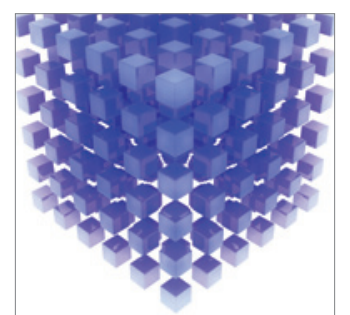

Mathematical Problems in Engineering
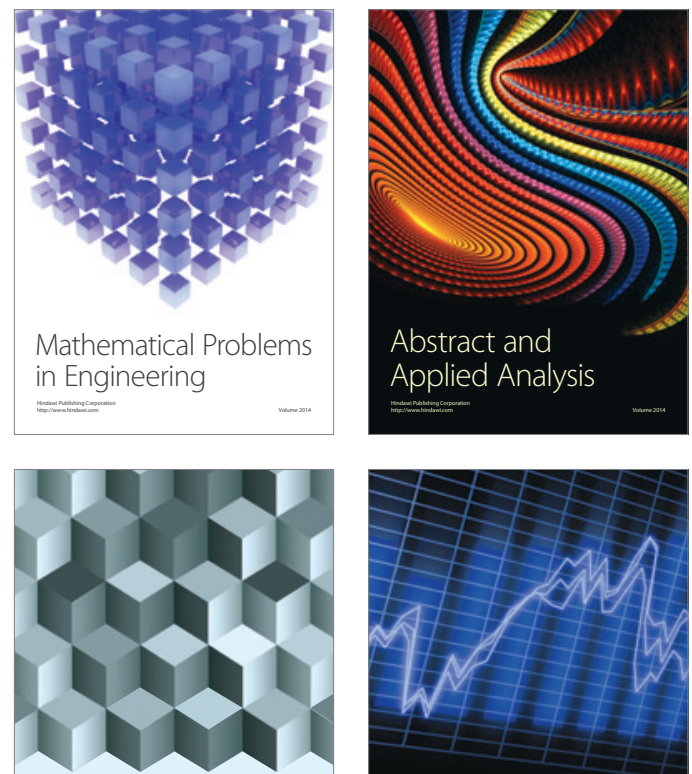

Journal of

Function Spaces

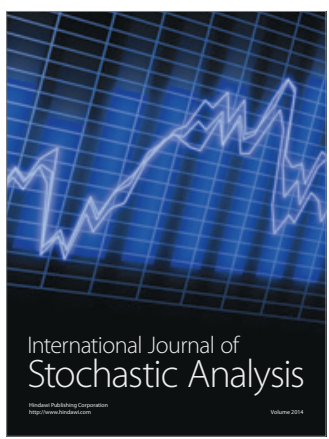

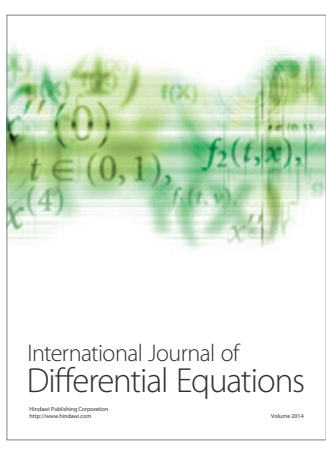
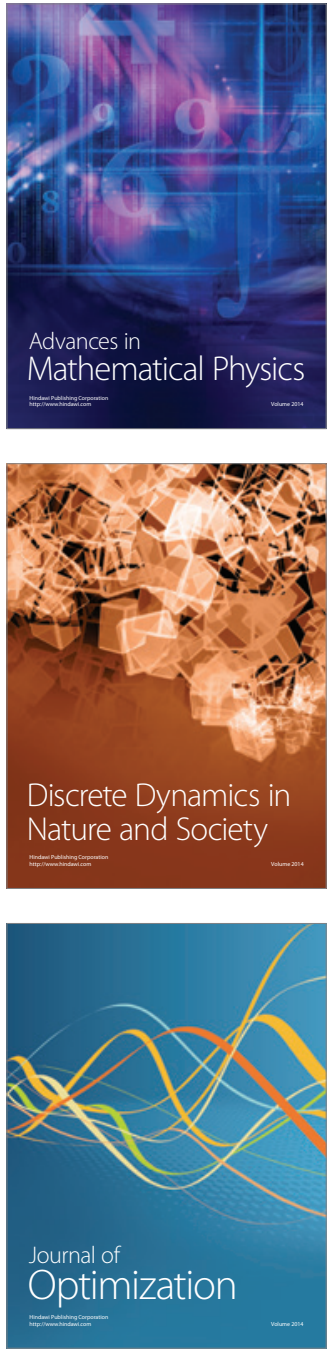